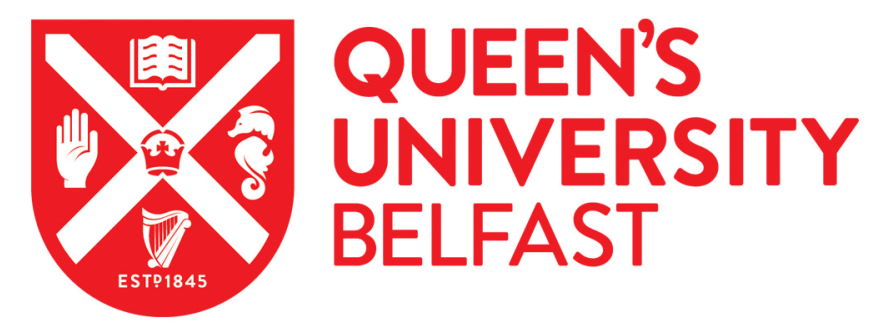

\title{
Dual Physically Cross-Linked k Carrageenan-Based Double Network Hydrogels with Superior Self-Healing Performance for Biomedical
} Application

Deng, Y., Huang, M., Sun, D., Hou, Y., Li, Y., Dong, T., Wang, X., Zhang, L., \& Yang, W. (2018). Dual Physically Cross-Linked $\mathrm{k}$ Carrageenan-Based Double Network Hydrogels with Superior Self-Healing Performance for Biomedical Application. ACS Applied Materials and Interfaces. https://doi.org/10.1021/acsami.8b15385

Published in:

ACS Applied Materials and Interfaces

Document Version:

Peer reviewed version

Queen's University Belfast - Research Portal:

Link to publication record in Queen's University Belfast Research Portal

Publisher rights

( 2018 American Chemical Society. This work is made available online in accordance with the publisher's policies. Please refer to any applicable terms of use of the publisher.

\section{General rights}

Copyright for the publications made accessible via the Queen's University Belfast Research Portal is retained by the author(s) and / or other copyright owners and it is a condition of accessing these publications that users recognise and abide by the legal requirements associated with these rights.

Take down policy

The Research Portal is Queen's institutional repository that provides access to Queen's research output. Every effort has been made to ensure that content in the Research Portal does not infringe any person's rights, or applicable UK laws. If you discover content in the Research Portal that you believe breaches copyright or violates any law, please contact openaccess@qub.ac.uk. 


\section{Dual Physically Cross-Linked $\kappa$-Carrageenan-Based Double Network Hydrogels with Superior Self- Healing Performance for Biomedical Application}

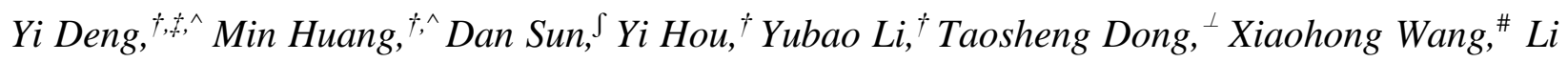
Zhang, ${ }^{* \dagger}$ Weizhong Yang ${ }^{*, \perp}$

${ }^{\dagger}$ Research Center for Nano-Biomaterials, Analytical and Testing Center, Sichuan University, Chengdu 610065, China

${ }^{\ddagger}$ School of Chemical Engineering, Sichuan University, Chengdu 610065, China

\#State Key Laboratory of Marine Resource Utilization in South China Sea, Hainan University, Haikou 570228, China

${ }^{\perp}$ College of Materials Science and Engineering, Sichuan University, Chengdu 610065, China

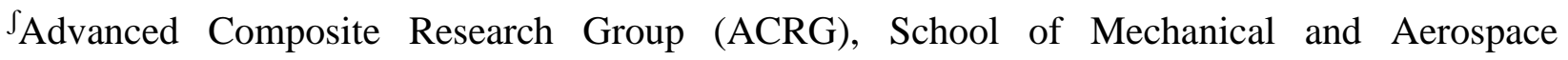
Engineering, Queens University Belfast, Belfast BT7 1NN, The United Kingdom 
ABSTRACT: Chemically linked Double network (DN) hydrogels display extraordinary mechanical attributes but mostly suffer from poor self-healing property and unsatisfactory biocompatibility due to the irreversible breaks in their chemical-linked networks and the use of toxic chemical crosslinking agents. To address these limitations, we developed a novel $\kappa-$ carrageenan/polyacrylamide (KC/PAM) DN hydrogel through a dual physical-crosslinking strategy, with the ductile, hydrophobically associated PAM being the first network, and the rigid potassium ion $\left(\mathrm{K}^{+}\right)$cross-linked $\mathrm{KC}$ being the second network. The dual physically cross-linked DN (DPC-DN) hydrogels with optimized KC concentration exhibit excellent fracture tensile stress $(1320 \pm 46 \mathrm{kPa})$ and toughness (fracture energy: $\left.6900 \pm 280 \mathrm{~kJ} / \mathrm{m}^{3}\right)$, comparable to those fully chemically linked DN hydrogels and physically-chemically cross-linked hybrid DN hydrogels. Moreover, owing to their unique dual physical-crosslinking structures, the KC/PAM hydrogels also demonstrated rapid self-recovery, remarkable notch-insensitivity, self-healing capability, as well as excellent cytocompatibility towards stem cells. Accordingly, this work presents a new strategy towards fabricating self-repairing DPC-DN hydrogels with outstanding mechanical behaviors and biocompatibility. The new type of DN hydrogels demonstrates strong potentiality in many challenging biomedical applications such as artificial diaphragm, tendon, and cartilage.

KEYWORDS: dual physically cross-linked, self-healing, mechanical property, biocompatibility, double network hydrogel. 


\section{INTRODUCTION}

Hydrogels are formed through the crosslinking of hydrophilic polymeric chains so that they can hold a significant amount of water. The water-rich feature of hydrogels has found widespread use in fields such as drug delivery, ${ }^{1-2}$ regenerative medicine, ${ }^{3-4}$ biosensor, ${ }^{5-6}$ cosmetics, ${ }^{7}$ and food chemistry. ${ }^{8-9}$ Nevertheless, the application of hydrogels in certain areas is still hindered due to their weak mechanical properties and/or lack of self-healing properties. Since Gong et al. used a twostep free-radical polymerization approach to create the first double network (DN) hydrogels in $2003,{ }^{10}$ DN hydrogels have captured increasing interests due to their appealing mechanical strength. DN hydrogels consist of two types of polymeric networks: (i) A stiff and brittle first network (e.g., bacterial cellulose,${ }^{11}$ agar, ${ }^{12}$ alginate ${ }^{13}$ ) that offers reversible sacrificial bonds and dissipates energy during the colossal deformation, and (ii) a soft and stretchable second network (e.g., poly(acrylic acid) (PAA) ${ }^{14}$ gelatin methacrylamide (GelMA) ${ }_{1}^{15}$ polyacrylamide (PAM) ${ }^{13}$ ), that endows hydrogels with elasticity. The unique network structure makes them both tough and soft, ${ }^{16}$ hence suitable for supporting and load-bearing material applications such as artificial cartilage, blood vessel, tendon, and diaphragm.

To date, most of the reported DN hydrogels are fully chemically cross-linked hydrogels, where both networks are covalently crosslinked. Their mechanical properties such as stiffness (elastic modulus 0.1-1.0 MPa), strength (tensile stress 1-10 MPa,strain 1000-2000 \%, compressive stress 30-60 MPa under strain 92-95\%), and toughness (fracture energy 100-1000 J/m²) are comparable to elastomer and natural cartilage. ${ }^{17}$ Despite their promising properties, the irreversible and permanent chain breakages in the first network under mechanical failture can severely obstruct their recovery and self-healing process. To enhance fatigue resistance and selfrecovery behaviors of DN hydrogels, researchers have introduced reversible noncovalent 
interactions containing ionic bond,,${ }^{13,18}$ hydrophobic association, ${ }^{12,19} \pi-\pi$ stacking, ${ }^{20}$ van der Waals force, ${ }^{21}$ host-guest interaction, ${ }^{22}$ and hydrogen bond ${ }^{23-24}$ to contruct the first network. In the physically-chemically linked hybrid DN hydrogels, the physical bonds in the first network will break firstly to dissipate energy under severe loading/deformation. When the material is unloaded, the physical bonds enable the hydrogels to reorganize and reconstruct, leading to the recovery of DN hydrogels. Following such design principle and synthesis methods, numerous physicallychemically cross-linked hybrid DN hydrogels such as agar/polyacrylamide, ${ }^{25}$ alginate/polyacrylamide, ${ }^{13,}{ }^{26}$ and bacterial cellulose/polyacrylamide, ${ }^{27}$ agar/polyvinyl alcohol ${ }^{28}$ have been fabricated. Although such hybrid DN hydrogels can dramatically boost the mechanical properties and self-recoverability, their fabrication techniques suffer from two main drawbacks: (1) The covalent crosslinked intrinsically soft and ductile second network (particularly PAAm-based DN hydrogels) cannot endure high loading condition after the first network is damaged. ${ }^{29}$ This normally leads to further rupture of the entire DN hydrogels. (2) The use of toxic chemical crosslinking agents for covalently-linked first and second networks usually compromise the biocompatibility of DN hydrogels. Therefore, development of dual physically cross-linked DN (DPC-DN) hydrogels that eliminate the use of toxic chemicals can potentially address these issues and lead to materials suitable for biomedical applications.

To date, only a few DPC-DN hydrogels with varying mechanical attributes and self-healing behaviors have been developed. Chen et al. fabricated agar/hydrophobically associated polyacrylamide (Agar/HPAM) DPC-DN hydrogels through a facile one-pot approach. Due to their unique fully physical and reversible bonds, the Agar/HPAM hydrogels displayed great tensile strength, toughness, as well as quick self-recovery and visible self-healing performance without external stimuli. ${ }^{25}$ Yuan and co-workers created a DPC-DN hydrogel using $\mathrm{Ca}^{2+}$ linked xanthan 
gum (XG) chains as the first network and hydrophobically associated PAM as the second network (called XG/PAM hydrogels). The tensile stress and compressive stress of the resultant hydrogels were $\sim 3.65 \mathrm{MPa}$ and $>50 \mathrm{MPa}$, respectively. ${ }^{18}$ Wang et al. developed poly(acrylic acid)$\mathrm{Fe}^{3+} /$ chitosan (PAA- $\mathrm{Fe}^{3+} / \mathrm{CS}$ ) DN hydrogels deploying metal-coordination and chain entanglement as the two means of physical crosslinking. The prepared hydrogels exhibited a fracture stress of 1.4 $\mathrm{MPa}$ and an elongation at rupture of $\sim 700 \%$ after self-recovery. ${ }^{30}$ Gong et al. prepared a poly(acrylamide-co-acrylic acid) (PAM-co-PAA)/poly(vinyl alcohol) (PVA) DN hydrogels through a facile two-step approach (copolymerization and freezing/thawing) to endow hydrogels with high mechanical, fatigue resistance, and self-recovery properties. ${ }^{31}$ Despite the promising results, few existing DPC-DN have demonstrated their extended applications in the biomedical field, mainly due to the lack of bioactive components for cell growth.

Carrageenan, one of the natural soluble polysaccharides derived from diverse species of marine red algae, possesses a linear sulfated galactan backbone with alternating $\alpha(1-3)$-D-galactose-4sulfated and $\beta(1-4)-3,6$-anhydro-D-galactose. ${ }^{32-34}$ Among different kinds of carrageenan (such as kappa, iota, lambda), $\kappa$-carrageenan $(\mathrm{KC})$ has a higher sensitivity to $\mathrm{K}^{+}$ions in forming hydrogels. ${ }^{35}$ Moreover, KC presents immunostimulatory and anti-tumor activity, ${ }^{36}$ and is proven to facilitate stem cell proliferation. ${ }^{37}$ As a result, it has been extensively used in tissue engineering, ${ }^{38-39}$ drug delivery ${ }^{40-41}$ and wound dressing. ${ }^{42}$ Herein, we design and fabricate a novel KC-based DPC-DN hydrogel consisting of hydrophobically associated PAM as the first network and ionic linked $\mathrm{KC}$ as the second network. We believe that the dual physical-crosslinking strategy will endow the hydrogels with the combined advantages of excellent mechanical property, selfrecovery, self-healing behaviors, as well as biocompatibility, which can significantly broaden the application of DN hydrogels, especially in the area of healthcare technologies. 


\section{EXPERIMENTAL SECTION}

\subsection{Materials}

-carrageenan $(\mathrm{KC})$ powder, acrylamide (AM) and stearyl methacrylate (SMA) were purchased from Shandong Xiya Chemical Industry Co., Ltd. (China). Sodium dodecyl sulfate (SDS), and ammonium persulfate (APS) were provided by Chengdu Kelong Reagent Factory (China). Potassium chloride $(\mathrm{KCl})$ was obtained from Beijing Kermel Co., Ltd. (China). All chemical reagents were used as received except that $\mathrm{KC}$ has been dried under $37 \square$ for $24 \mathrm{~h}$ before use.

\subsection{Fabrication of KC/PAM DPC-DN Hydrogels}

$\mathrm{KC}(0.489,0.978,1.434$ or $1.956 \mathrm{~g})$ was first dissolved in $8 \mathrm{wt} \% \mathrm{SDS} / 0.9 \mathrm{M} \mathrm{NaCl}$ water solution $(50 \mathrm{~mL})$ and stirred for $2 \mathrm{~h}$ under $70{ }^{\circ} \mathrm{C}$. After the homogeneous solution was cooled down to $45^{\circ} \mathrm{C}$, AM (19.56 g) and SMA (1.87 g, 2 mol\% of AM) were introduced, and the resulting solution was stirred for $60 \mathrm{~min}$. To obtain the second network, $\mathrm{KCl}$ (6 wt $\%$ of $\mathrm{KC}$ weight) and APS (0.6 wt $\%$ of AM weight) were added into the solution, followed by rapid degassing and transferring into a glass mold $(\Phi 50 \mathrm{~mm} \times 2 \mathrm{~mm})$ in an inert atmosphere. The sample was kept at $70{ }^{\circ} \mathrm{C}$ for 2 days to complete the polymerization process. The prepared DPC-DN hydrogels were named as KC/PAM$\mathrm{n}$, where $\mathrm{n}$ represents the weight $\%$ of $\mathrm{KC}$ relative to $\mathrm{AM}(\mathrm{n}=0,2.5,5,7.5,10)$. The PAM SN hydrogels were prepared by the same method without addition of KC. AM (19.56 g) and SMA (1.87 g) were dissovled in $8 \mathrm{wt} \% \mathrm{SDS} / 0.9 \mathrm{M} \mathrm{NaCl}$ solution at $45^{\circ} \mathrm{C}$ and stirred for $60 \mathrm{~min}$. Then, APS (0.1174 g) was added into the solution followed by degassing and molding. The resulting materials were stored at $70{ }^{\circ} \mathrm{C}$ in an inert atmosphere for 2 days to complete polymerization and form PAM SN hydrogels. The KC SN hydrogels were prepared by dissolving an appropriate 
amount of $\mathrm{KC}$ powders into $\mathrm{KCl}\left(6 \mathrm{wt} \%\right.$ of $\mathrm{KC}$ weight) solution and agitated for $2 \mathrm{~h}$ under $70^{\circ} \mathrm{C}$ After cooling, the KC SN hydrogels were formed.

\subsection{Characterization}

Fourier transform infrared (FT-IR) spectrometer (Nicolet 6700, Thermo) was employed to detect the chemical constitute of the KC/PAM hydrogels. The microstructures of freeze-dried and cryogenically fractured hydrogel surfaces were analyzed using with a scanning electron microscope (SEM; S4800, Hitachi, Japan), and the pore size distribution was analysis using ImageJ software. All fractured surfaces were coated with Au before measurement. Dynamic thermomechanical analysis (DMA) was conducted using DMA Q800 instrument (TA Instruments, USA). samples ( $4 \mathrm{~mm}$ in diameter and $4 \mathrm{~mm}$ in thickness) were subjected to sinusoidal deformation (amplitude $=20 \mu \mathrm{m}$ ) from $25^{\circ} \mathrm{C}$ to $70^{\circ} \mathrm{C}$ at a heating rate of $2{ }^{\circ} \mathrm{C} / \mathrm{min}$. The equilibrium water contents and water swelling ratio of KC/PAM hydrogels were evaluated gravimetrically. Specially, the cylindrical hydrogel samples were immersed in D.I. water. The swollen hydrogels were weighed after reaching swelling equilibrium. After that, the samples were dried at $60^{\circ} \mathrm{C}$ until no further weight loss. The water contents and swelling ratio of hydrogels were determined according to the following equations:

$$
\begin{array}{ll}
\text { Equilibrium water content }=\frac{W_{1}-W_{2}}{W_{1}} \times 100 \% & \text { Eq (1) } \\
\text { Swelling ratio }=\frac{W_{1}}{W_{0}} \times 100 \% & \text { Eq (2) }
\end{array}
$$

where $W_{0}$ is the weight of the as-prepared hydrogels, $W_{l}$ is the weight of the swollen hydrogels, $W_{2}$ is the weight of the dry cylindrical hydrogels.

\subsection{Mechanical Testing}

The hydrogels were cut to dumbbell-shaped samples $(75 \mathrm{~mm} \times 4 \mathrm{~mm} \times 1.8 \mathrm{~mm})$, and tested using a universal testing machine (AGIC 50KN, Shimadzu, Japan) following previously established 
protocols. ${ }^{43}$ The samples were stretched at a speed of $50 \mathrm{~mm} / \mathrm{min}$ under a tensile load of $10 \mathrm{~N}$ under ambient condition. The tensile strength and elongation of hydrogels at rupture were investigated. The Young's modulus of the hydrogels was determined according to the slope of the stress-strain curve. To investigate the energy dissipation mechanism, the dumbbell-shaped samples were stretched to a given stretch ratio $(\lambda)$ and unloaded instantly to $0 \mathrm{~N}$ at a rate of $50 \mathrm{~mm} / \mathrm{min}$. The dissipated energy ( $U_{\text {hys }}$ ) was calculated by the area below the stress-strain curves. Each sample was tested 5 times, and the average value was used for further analysis.

\subsection{Evaluation of Self-Healing Properties}

To measure the self-healing properties, round disc specimens of hydrogels were cut into halves, and one of the discs was doped with rhodamine B for better observation. The cut discs with different colors were joined together at the cut surface and were sealed in a polyethylene (PE) bag to prevent water evaporation. Then, samples were divided into groups and stored at different temperatures $\left(37^{\circ} \mathrm{C}, 50^{\circ} \mathrm{C}\right.$ and $\left.70{ }^{\circ} \mathrm{C}\right)$ for different periods of time $(2,5,12,18$ and $24 \mathrm{~h}$, respectively). After healing, the tensile tests were carried out at $50 \mathrm{~mm} / \mathrm{min}$, and 5 tests were carried out for each sample.

\subsection{Isolation and Culture of Primary rBMSCs from Rats}

Experiments involving animals have been approved by the Institutional Animal Care and Use Committee (IACUC) of the West China Hospital, Sichuan University. Briefly, the rats were sacrificed, the metaphysis from both ends of the femurs was cut open, and the marrow was extracted using low-glucose $\alpha$-MEM (Sigma-Aldrich, USA). The isolated rat bone marrowderived mesenchymal stem cells (rBMSCs) were then cultivated in the $\alpha$-MEM media with $10 \%$ fetal calf serum (Hyclone, USA), $1 \%$ penicillin-streptomycin (Gibco), and then cultured in an incubator (Thermo, USA) under $5 \% \mathrm{CO}_{2}$ at $37^{\circ} \mathrm{C}$. The medium was refreshed every 2 days, and 
cells from passage 3 were used for further investigation.

\subsection{Cell Viability Test}

Viabilities of rBMSCs were tested using cell counting kit-8 assay (CCK-8, Dojindo, Japan). After counting, rBMSCs were re-seeded onto pristine PAM and KC/PAM hydrogels with a density of $2 \times 10^{5} / \mathrm{mL}$. After culturing for different periods of time (1-5 days), the CCK- 8 kit was supplemented into each well at a ratio of 1:10 for $2 \mathrm{~h}$ dark incubation. Afterwards, $100 \mu \mathrm{L}$ of supernatant from each well was transferred to a new 96-well plate. The average optical density (OD) of the supernatant was recorded using the microplate reader (VariOskan Flash 3001, USA) at $450 \mathrm{~nm}$. Six samples were tested for each group to ensure the statistical significance.

\subsection{SEM and Cytoskeletal Observation of rBMSCs}

FE-SEM was used to observe the cell morphology grown on the materials. After incubation for 1 day, cells on the samples were cleaned with PBS and fixed using $2.5 \mathrm{w} / \mathrm{v} \%$ glutaraldehyde for $3 \mathrm{~h}$. The samples were then dehydrated with a series of alcohol solutions (concentrations ranging from $30 \%$ to $100 \%, 20$ min for each concentration). Samples were dried using critical point drying followed by gold sputter coating for SEM observation (S4800).

rBMSCs were washed with a buffer solution and fixed using $4 \mathrm{w} / \mathrm{v} \%$ paraformaldehyde for 0.5 $\mathrm{h}$ at $24 \mathrm{~h}$ of culture. The cells were then permeabilized with $0.1 \%$ Triton X-100 for $8 \mathrm{~min}$, before incubation in $0.25 \% \mathrm{BSA} / \mathrm{PBS}$ solution for $20 \mathrm{~min}$ to block non-specific binding. Then, $10 \mu \mathrm{g} / \mathrm{mL}$

of rhodamine B-labeled phalloidin was used to stain cells for $0.5 \mathrm{~h}$ at room temperature. After rinsing with buffer, cells were further stained by $8 \mu \mathrm{g} / \mathrm{mL}$ DAPI (Sigma-Aldrich) for $20 \mathrm{~min}$. The fluorescent signal from cells was captured using confocal laser scanning microscopy (CLSM, LSM510, Germany).

\subsection{Statistical Analysis}


Statistical analysis was performed through SPSS 19.0 software to determine the significant differences among the groups, and $p$ values $<0.05$ were regarded indicative of statistical significance.

\section{RESULTS AND DISCUSSION}

\subsection{Fabrication of KC/PAM DPC-DN Hydrogels}

Figure 1. presents the schematic diagram for the preparation of the novel KC-based DPC-DN hydrogels, where different types of physical crosslinking bonds have been highlighted. SMA is dissolved in the SDS micelles to generate polymerizable micelles. After that, a physically crosslinked first network of poly(AM-co-SMA) is formed through the micelle copolymerization of AM and SMA. During cooling, $\kappa$-carrageenan chains undergo a coil-helix transition. The further agglomeration of single helices leads to the formation of double helices through (i) ionic bonding between the $\mathrm{K}^{+}$ion and the sulfate group of d-galactose, and (ii) an electrostatic interaction between the $\mathrm{K}^{+}$ion and the anhydro-O-3, 6 ring of another d-galactose residue. ${ }^{35}$ This process results in the formation of the physically linked KC second network. The first network, which interpenetrates the second $\mathrm{KC}$ network, is mainly associated with strong hydrophobic interactions between SDS micelles and the hydrophobic alkyl groups of SMA. Also, hydrogen bonds are present between PAM and KC networks. Distictively different from other well reported chemically linked and hybrid-linked DN hydrogels, the dual physically cross-linked KC/PAM DN hydrogel we developed has no chemical crosslinking in both networks. 


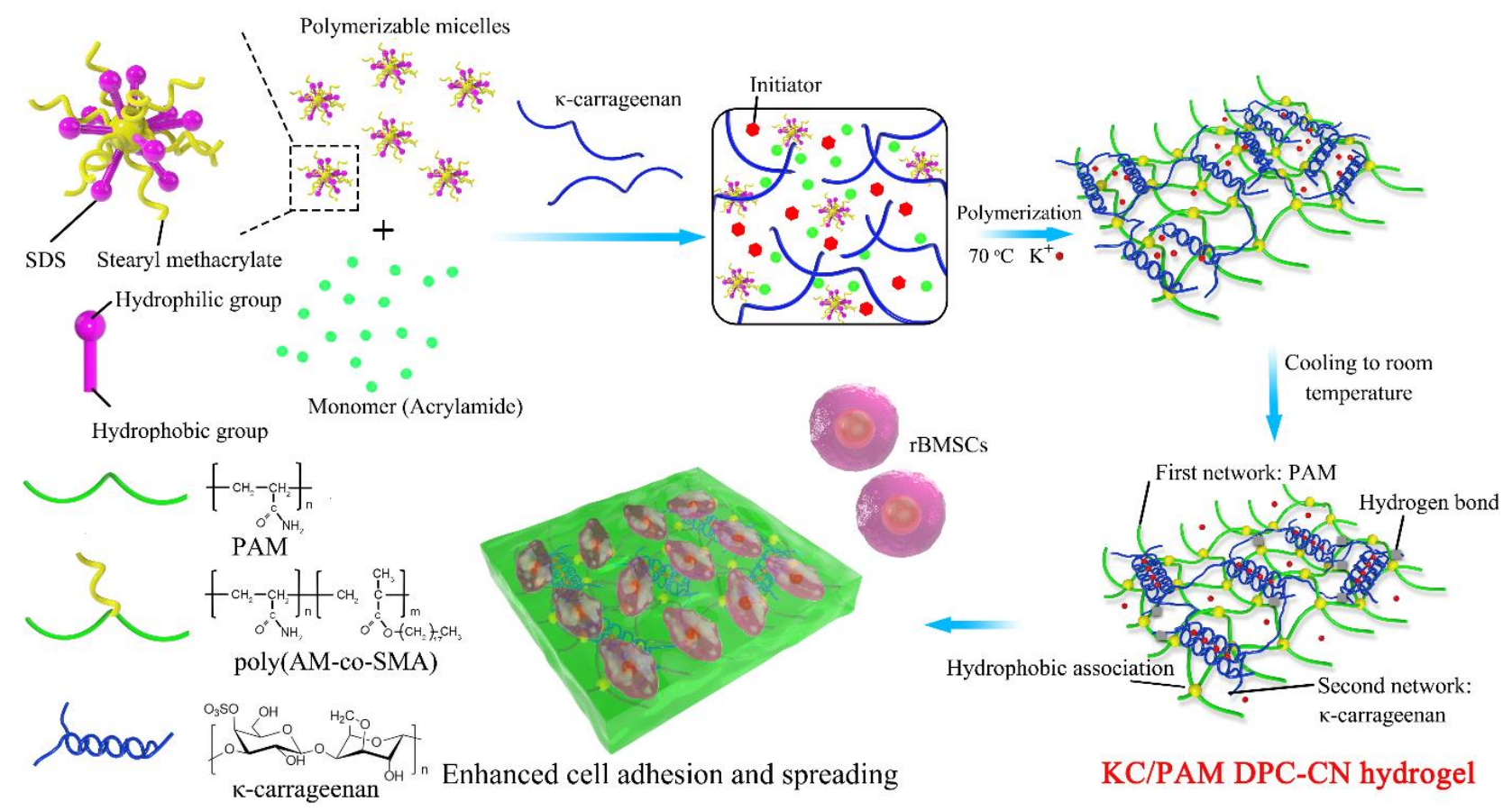

Figure 1. Schematic drawing of the fabrication of the KC/PAM DPC-DC hydrogel with its proposed network structures and the cell responses on the hydrogel.

Figure 2 shows the FT-IR spectra of KC, PAM, and a series of KC/PAM DPC-DN hydrogels. The characteristic peaks of $\mathrm{KC}$ at $1379,1048,933$ and $859 \mathrm{~cm}^{-1}$ correspond to sulfate ester, glycosidic linkage, ${ }^{44}$ and 3, 6-anhydro-D-galactose and D-galactose-4-sulfate, respectively. ${ }^{45} \mathrm{~A}$ peak at $1157 \mathrm{~cm}^{-1}$ is associated with the stretching vibration of the $\mathrm{C}-\mathrm{O}-\mathrm{C}$ group. The PAM hydrogel exhibits apparent peaks at $3415 \mathrm{~cm}^{-1}$ and $3198 \mathrm{~cm}^{-1}$ for a stretching vibration of N-H. The peaks at $1664 \mathrm{~cm}^{-1}$ and $1614 \mathrm{~cm}^{-1}$ can be assigned to $\mathrm{C}=\mathrm{O}$ stretching, and $\mathrm{N}-\mathrm{H}$ deformation of the primary amine, respectively. ${ }^{13}$ In comparison, apart from the characteristic peaks corresponding to $\mathrm{KC}$ and PAM, no new adsorption peak emerged from the FT-IR spectra of $\mathrm{KC} / \mathrm{PAM}$ hydrogel, implying there is no new chemical bonds formation in the DN structure. Nonetheless, there is a shift of glycosidic linkage in $\mathrm{KC}$ from $1048 \mathrm{~cm}^{-1}$ to $1033 \mathrm{~cm}^{-1}$ in KC/PAM hydrogel, which may result from the formation of intermolecular hydrogen bonds between $\mathrm{KC}$ and PAM polymers. 


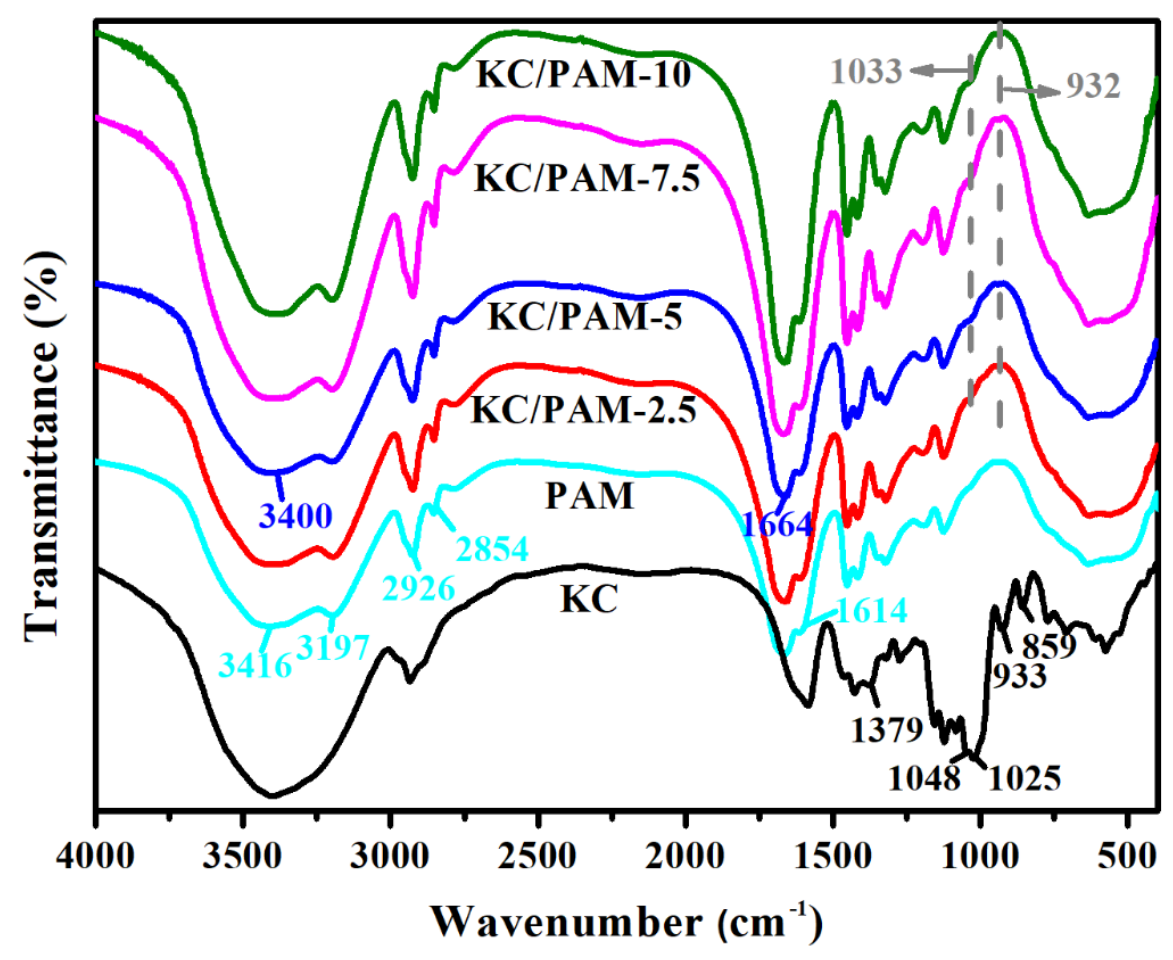

Figure 2. FT-IR spectra of pristine KC, PAM, and KC/PAM hydrogels.

The microstructures of the cryo-fractured pristine $\mathrm{KC}$, PAM and KC/PAM hydrogels were analyzed using SEM after lyophilization. The pores size of $\mathrm{KC}$ hydrogel (Figure 3a) are random. In contrast, PAM hydrogel features generally larger pores with an average size of approx. $120 \mu \mathrm{m}$ and the walls of the macropores appear to be thinner. The KC/PAM DPC-DN hydrogels exhibit distinctive morphologies because of the double network. It is apparent that with increasing amount of $\mathrm{KC}$, the pore size of $\mathrm{KC} / \mathrm{PAM}$ hydrogels decreases and the pore size distribution becomes tighter, suggesting the formation of a denser structure, as shown in Figure 3c-f and Figure S1. 

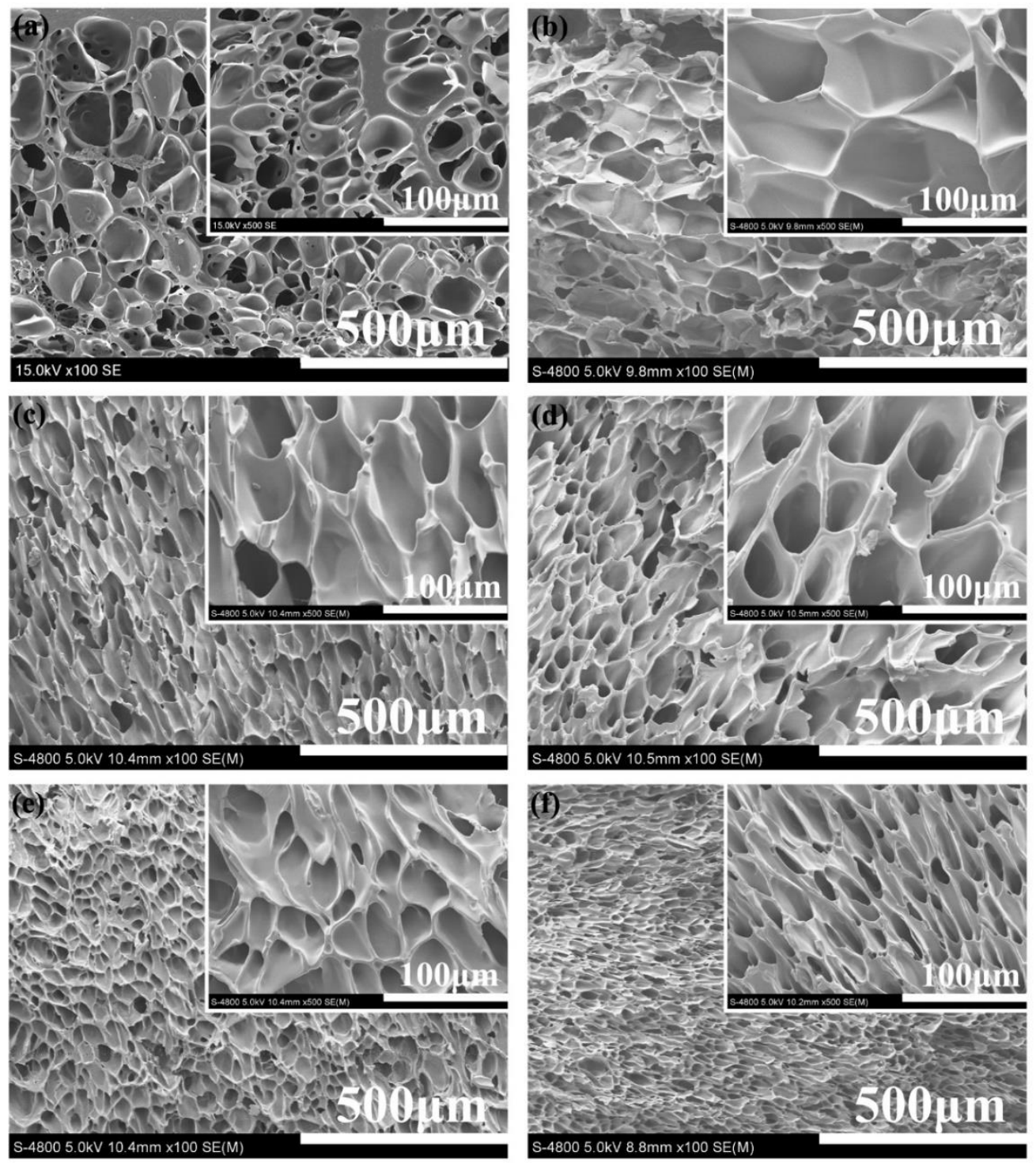

Figure 3. The SEM images of (a) pristine KC, (b) PAM, (c) KC/PAM-2.5, (d) KC/PAM-5, (e) KC/PAM-7.5, and (f) KC/PAM-10 hydrogels.

The equilibrium water contents and swelling ratio of various KC/PAM DPC-DN hydrogels have been measured. Pristine PAM hydrogels contain about $95.5 \%$ of water. The equilibrium water contents of $\mathrm{KC} / \mathrm{PAM}$ hydrogels is at a similar level for samples containing $0-5 \% \mathrm{KC}$ but the value starts to decrease with further increase in $\mathrm{KC}$ concentration (Figure S2a). The main reason is that the formation of the DN network densifies the hydrogel structure and thickens the pore walls, hence hinders the penetration of water molecules. Figure S2b reveals that the swelling ratios of all 
hydrogels increase rapidly in the first $75 \mathrm{~h}$ followed by a more gradual increase afterwards. In addition, the swelling ratio of the hydrogels increases with the increasing $\mathrm{KC}$ content.

The TG-DTG curves of all KC/PAM-10 hydrogels are presented in Figure S3. The initial weight loss of all samples below $150{ }^{\circ} \mathrm{C}$ might be ascribed to the evaporation of hydrogen bound water. The significant weight loss of $\mathrm{KC}$ samples ranging from $210{ }^{\circ} \mathrm{C}$ to $700{ }^{\circ} \mathrm{C}$ results from the degradation of molecular chains. ${ }^{46}$ The pristine PAM hydrogels display a multistep thermolysis. The degradation that takes place between $230{ }^{\circ} \mathrm{C}$ and $340{ }^{\circ} \mathrm{C}$ can be attributed to the reaction between two amide groups, which lead to the formation of a single amide group and release of the ammonia molecule. ${ }^{47}$ The third weight loss in the range of $340-500{ }^{\circ} \mathrm{C}$ is caused by the disintegration of imides and the PAM backbone. ${ }^{48}$ Similar to the PAM hydrogel, the degradation of KC/PAM-10 hydrogel also follows three stepsbut with a greater residual mass. The peak decomposition rate (Figure S3) of KC/PAM hydrogels in the second thermolysis process also shifts to a higher temperature. These results indicate that KC/PAM DPC-DN hydrogels display a better thermal stability than PAM, due to the existence of intermolecular hydrogen interactions between the $\mathrm{KC}$ and PAM macromolecules as discussed earlier.

\subsection{Mechanical Characterization of KC/PAM DPC-DN Hydrogels}

The mechanical properties including mechanical strength, stretchability, and notch-insensitivity of the pristine PAM and KC/PAM DPC-DN hydrogels were evaluated systematically. The tensile stress-strain curves of KC/PAM hydrogel specimens are shown in Figure 4a. Due to the presence of SDS micelles, the elongation at rupture for pristine PAM hydrogels reaches $970 \%$ at a tensile stress of $118 \mathrm{kPa}$, far exceeding the traditional chemically linked PAM single network (SN) hydrogels (elongation at rupture: $660 \%$, tensile stress: $11 \mathrm{kPa}$ ). ${ }^{13}$ In contrast, the KC/PAM DPCDN hydrogels show gradually increased mechanical properties, see Figure 4a-c. The greatest 
elongation at rupture $(1400 \%)$ and tensile strength $(1350 \mathrm{kPa})$ are recorded for KC/PAM-10 hydrogels. Furthermore, Young's modulus and fracture energy of KC/PAM-10 hydrogels also show drastic improvements $\left(180 \mathrm{kPa}\right.$ and $\left.6926 \mathrm{~kJ} / \mathrm{m}^{3}\right), 3.2$ times and 13.6 times higher than those of pristine PAM hydrogels (57 kPa and $510 \mathrm{~kJ} / \mathrm{m}^{3}$, respectively, see Figure $4 \mathrm{~b}-\mathrm{c}$ ). In addition, our KG/PAM-10 DPC-DN are comparable to tensile properties of several fully chemically crosslinked DN hydrogels such as poly(2-acrylamide-2-methyl-propane sulfonic acid)/PAM, bacterial cellulose/poly( $N, N^{\prime}$-dimethyl acrylamide), gelatin/cellulose DN hydrogels. ${ }^{17,49} \mathrm{We}$ also find that the tensile strength, Young's modulus and fracture energy all improve with the increasing KC concentration in hydrogels, indicating that the PAM hydrogel can be remarkably reinforced by the second network made of ionically cross-linked KC chains.
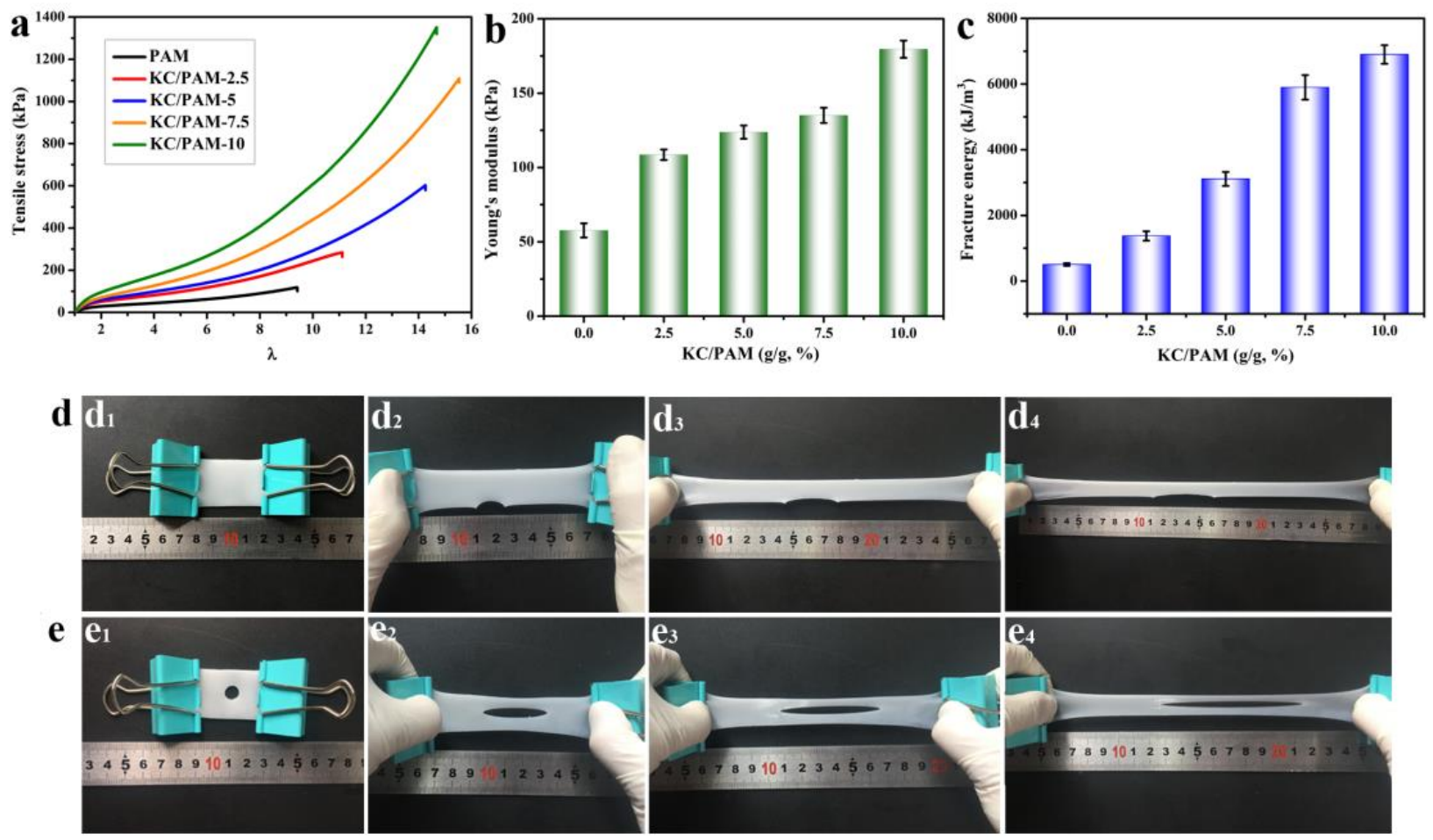

Figure 4. (a) Tensile stress-strain curves, (b) Young's modulus, and (c) fracture energy of the pristine PAM and KC/PAM DPC-DN hydrogels with varying KC content. The photographs of notch-sensitivity tests of KC/PAM-10 hydrogels with (d) single-edge notch and (e) central notch. 
We further investigated the crack resistance of KC/PAM DPC-DN hydrogel through stretching two-notched samples (40 $\mathrm{mm} \times 35 \mathrm{~mm})$, namely (i) single-edge notched $(\Phi 8 \mathrm{~mm})$ and (ii) central notched $(\Phi 8 \mathrm{~mm}) \mathrm{KC} / \mathrm{PAM}-10$ hydrogels. As is shown in Figure 4d-e, both samples remain stable without crack propagation even when the stretch ratio reaches 6.5 and 7 , respectively. It was reported by Gong et al. that once the ratio of $\sigma_{\mathrm{f}} / \mathrm{E}$ is greater than 2 (where $\sigma_{\mathrm{f}}$ and $\mathrm{E}$ denote fracture stress and modulus, respectively), crack blunting will occur. ${ }^{50}$ The $\sigma_{\mathrm{f}} / \mathrm{E}$ values of KC/PAM-n hydrogels prepared in this work are between 2.61-7.50, indicating their insensitivity to notches and hence they are immune to crack propagation. Such phenomena might be due to the synergistic effects of the hydrophobically associated PAM first network and the KC second network. When a notched DPC-DN hydrogel is stretched, the KC network dissipates and transfers the energy around the crack to the neighboring area. Simultaneously, the PAM network bridges cracks and stabilizes the deformation, removing the stress concentration to prevent crack propagation.

\subsection{Self-Recovery and Self-Healing Performance of KC/PAM DPC-DN Hydrogels}

Considering that both networks in KC/PAM DPC-DN hydrogels are physically cross-linked through reversible noncovalent interactions, cyclic loading measurements were further conducted to assess energy dissipation capacity and self-recovery property of KC/PAM hydrogels at room

temperature. Figure 5a-b shows the dissipated energy ( $\left.\mathrm{U}_{\text {hys }}\right)$ of KC/PAM-10 hydrogels as a function of the stretch ratio $(\lambda)$. The peak dissipated energy $\left(823 \mathrm{~kJ} / \mathrm{m}^{3}\right)$ at $\lambda=5$ is much greater than that for physically linked PAM hydrogels reported in the literature $\left(86 \mathrm{~kJ} / \mathrm{m}^{3}\right) .{ }^{25}$ At $\lambda=6$, KC/PAM hydrogels display a bigger hysteresis hoop, while PAM hydrogels have a minimal hysteresis under the same conditions (Figure 5c). Moreover, the DMA results in Figure S4 reveal that for $\mathrm{KC} / \mathrm{PAM}-10$, the storage modulus $\mathrm{G}^{\prime}$ is higher than the loss modulus $\mathrm{G}$ " even at an elevated temperature. This confirms that the hysteresis during the cyclic loading/unloading process 
results from the rupture of the physical bonds rather than from chemically covalent bonds. ${ }^{32}$ These results suggest that KC/PAM DPC-DN hydrogels can dissipate energy more effectively than the pristine PAM hydrogels upon deformation. However, smaller hysteresis loops shown by the 2nd and 3rd re-loading indicate that the network cannot recover immediately.
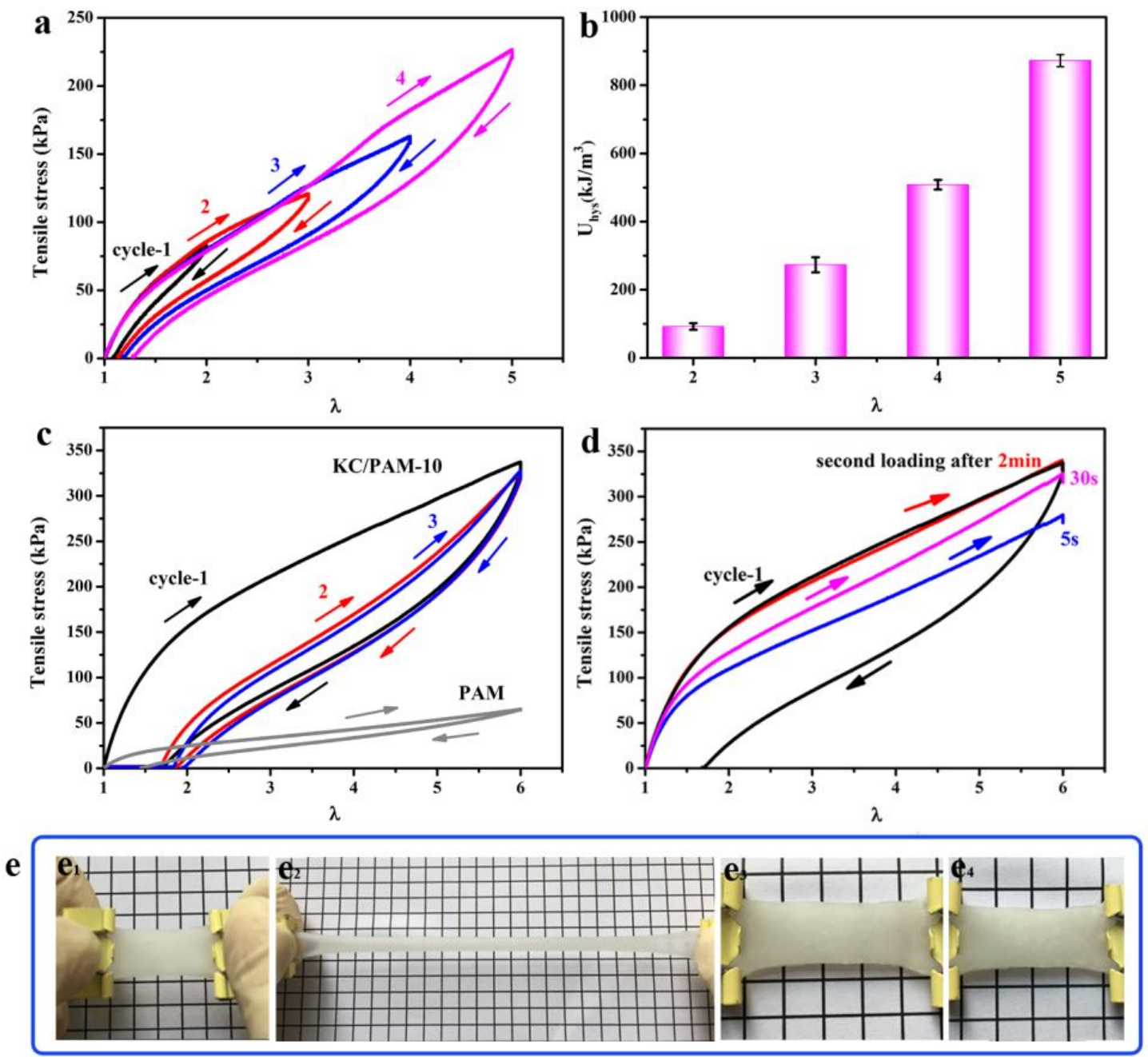

Figure 5. (a) Loading/unloading stress-strain curves and (b) the dissipation energy of KC/PAM10 hydrogels at different stretch ratios, (c) Loading/unloading stress-strain curves of KC/PAM-10 hydrogels at $\lambda=6$, (d) Recovery curves of KC/PAM-10 hydrogels after different relaxation time after the first loading/unloading cycle, (e) KC/PAM-10 hydrogels in (e1) original length and (e2) 
after being stretched at $\lambda=6$; KC/PAM-10 hydrogels recovered from stretching for (e3) $0 \mathrm{~s}$ and (e4) 2 min.

To better understand the recovery performance of KC/PAM-10 hydrogels, the hydrogel samples were stretched to $\lambda=6$. The force is then released, and samples were stored in a sealed PE bag under ambient temperature for different periods of time, followed by cyclic loading measurements. As shown in Figure 5d, the hysteresis loop almost recovers to its original state after 2 min sample storage. The recovery process of KC/PAM hydrogel is shown in Figure 5e. Ratio $\sigma_{2} / \sigma_{1}$ (where $\sigma_{1}$ and $\sigma_{2}$ denote the elastic modulus in the first and second loading cycle, respectively) was used as an index to characterize the hydrogel recovery efficiency (elasticity). It can be seen that revovery rates for stressed hydrogels with 5s and 2 min relaxation time are $83 \%$ and $100 \%$, respectively. In the previous literature, Liu et al. achieved $100 \%$ recovery of hybrid cross-linked KC/PAM at $90{ }^{\circ} \mathrm{C}$ and claimed that the thermoreversible sol-gel transition $\left(50{ }^{\circ} \mathrm{C}-60{ }^{\circ} \mathrm{C}\right)$ of $\mathrm{KC}$ was responsible for the recovery of $\mathrm{KC} / \mathrm{PAM}$ hydrogels. ${ }^{32}$ However, our fully physically cross-linked KC/PAM DN hydrogels exhibit a near $100 \%$ recovery at room temperature, which can be ascribed to the synergistic effect of the interpenetrating double networks. During the first stretching, the fracture of double-helix aggregates of $\mathrm{KC}$ proceeds by unzipping ionic crosslinks and pulling out the chains ("ion-pulling-out" fracture mechanism). The $\mathrm{KC}$ double helices gradually migrated from the $\mathrm{K}^{+}$induced KC helices bundles. Such "ion-pulling-out" and dissociation performance of $\mathrm{KC}$ neither rupture the second network nor alter $\mathrm{KC}$ helix conformation, hence the $\mathrm{KC}$ network still maintains intact within the hydrogels. Once the external force is removed, $\mathrm{K}^{+}$ions rapidly revert to their initial positions and reunite with the second network through reformation of the double helix domains. Since the first network is made of the reversible and noncovalent bonds through a strong hydrophobic interaction between SDS micelles and the alkyl groups of SMA side 
chains, the temporary dissociation of noncovalent bonds in the first PAM network can be recovered at ambient temperature with no external stimuli. Hence, the synergistic effect of the first network and second network can dramatically dissipate energy when the DPC-DN hydrogels are subject to external force or deformation.

Apart from self-recovery experiments using intact hydrogels, we also tested the KC/PAM DPCDN hydrogels for their self-healing properties. Figure 6a shows a typical sample (KC/PAM-2.5 hydrogel) that exhibited strong healing after $8 \mathrm{~h}$ of joining. Figure $6 \mathrm{~b}$ further presents the tensile stress-strain curves of original and self-healed DPC-DN hydrogels. Despite showing exactly the same stress-strain behavior at the initial state, the self-healed DPC-DN hydrogels demonstrated a smaller elongation at break across the range of samples. The healing efficiency (defined by $\sigma_{\text {self- }}$ healed $\left./ \sigma_{\text {original }}\right)$ of PAM and $\mathrm{KC} / \mathrm{PAM}$ hydrogels based on the stress-strain results are presented in Figure $6 \mathrm{c}$. The healing efficiencies decrease from $57 \%$ for PAM to $33 \%$ for KC/PAM-10 hydrogels. Although the elastic moduli of the self-healed hydrogels are comparable to those of the uncut samples, their strength and toughness decreased. The possible reason might be that the fractured chains cannot revert to their original state. Moreover, the $\mathrm{KC}$ concentration, time and temperature can significantly influence the self-healing efficiency of KC/PAM DPC-DN hydrogels. The self-healing efficiency of KC/PAM DN hydrogels decreases with increasing $\mathrm{KC}$ since $\mathrm{KC}$ might restrict the movement of molecular chains. In addition, as shown in Figure 6d, the selfhealing efficiency increases significantly over time, i.e., from $\sim 33 \%$ to $\sim 49 \%$ after $24 \mathrm{~h}$ at $70{ }^{\circ} \mathrm{C}$ for KC/PAM-2.5 hydrogels. The self-healing efficiency also increases with increasing temperature, as is shown in Table S1). On one hand, the elevated temperature can soften the hydrogels and allow for better contact between the fractured surfaces. One the other hand, the self-healing can be accelerated due to the increased chain mobility crossing the cut surface under higher temperature, 
especially at the temperature above the $\mathrm{KC}$ sol-gel transition temperature. Such results suggest that the employment of the physically cross-linked PAM as the first network endows the DN hydrogels with excellent self-healing behaviors.
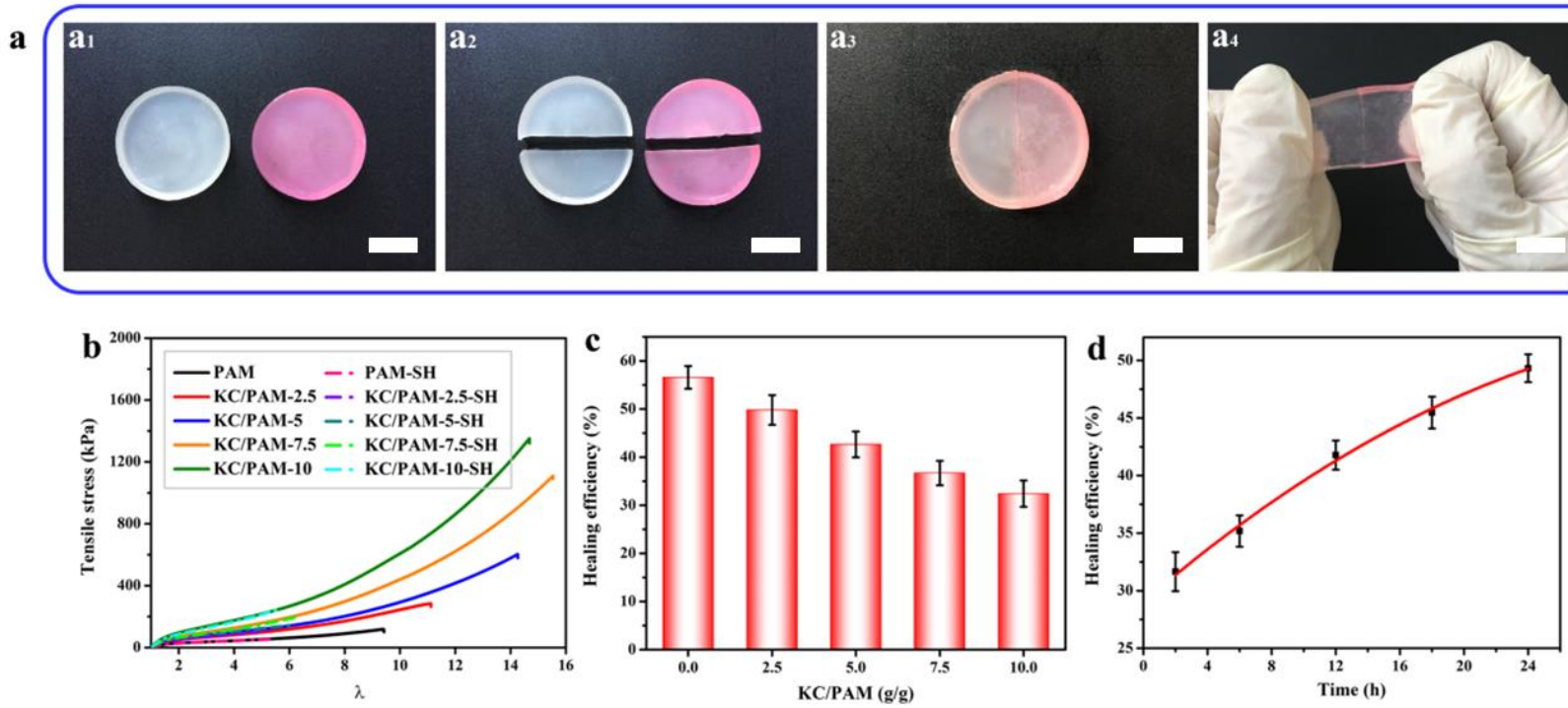

Figure 6. (a) Self-healing behavior of KC/PAM-2.5 hydrogels: (a $\left.a_{1}\right)$ two original hydrogel discs with or without dye; $\left(a_{2}\right)$ freshly cut hydrogels in halves; $\left(a_{3}\right)$ the cut hydrogel (halves) from different samples being joint together at $70{ }^{\circ} \mathrm{C}$ for $24 \mathrm{~h} .\left(\mathrm{a}_{4}\right)$ the cut hydrogel (two halves) healed after $8 \mathrm{~h}$ of storage. (b) Tensile stress as a function of stretch ratio for the original samples (solid lines) and self-healed samples (dash lines) at $70{ }^{\circ} \mathrm{C}$ after $24 \mathrm{~h}$. (c) Healing efficiency of corresponding self-healed DN hydrogels in (b). (d) Healing efficiency of KC/PAM-2.5 hydrogel healed after various periods of time at $70{ }^{\circ} \mathrm{C}$. Scale bar: $2 \mathrm{~cm}$.

\subsection{Mechanism of Self-Healing Properties}

Figure 7 describes the potential self-healing mechanism of the novel KC/PAM DPC-DN hydrogels. When the KC/PAM hydrogels are cut into halves, both the $\mathrm{KC}$ helices bundles in the second network and the hydrophobic association in the first network are disterburbed/broken. With the presence of water within the hydrogel, the SDS present on the fractured surfaces dissociatesand 
decreases the surface tension of the cut surface effectively. After the cut pieces are joint together, with the evaporation and infiltration of water, a local, high SDS concentration drives the disassembled SDS to reassemble with stearyl group of SMA in the side chains of PAM, leading to reformation of the hydrophobic agglomerations. Besides, when the samples are heated to $70{ }^{\circ} \mathrm{C}$ (the temperature above the sol-gel transition temperature), the $\mathrm{KC}$ double helices are disentangled into a single coil. As the temperature decreases, single coils transform into single helices through the coil-helix transition. Then with the help of $\mathrm{K}^{+}$ions, they further assemble and recover to the double-helical aggregates. Therefore, the hydrophobic association and $\mathrm{K}^{+}$-induced helices bundle formation both contribute to the superior self-healing behavior of the novel KC/PAM DPC-DN hydrogels.

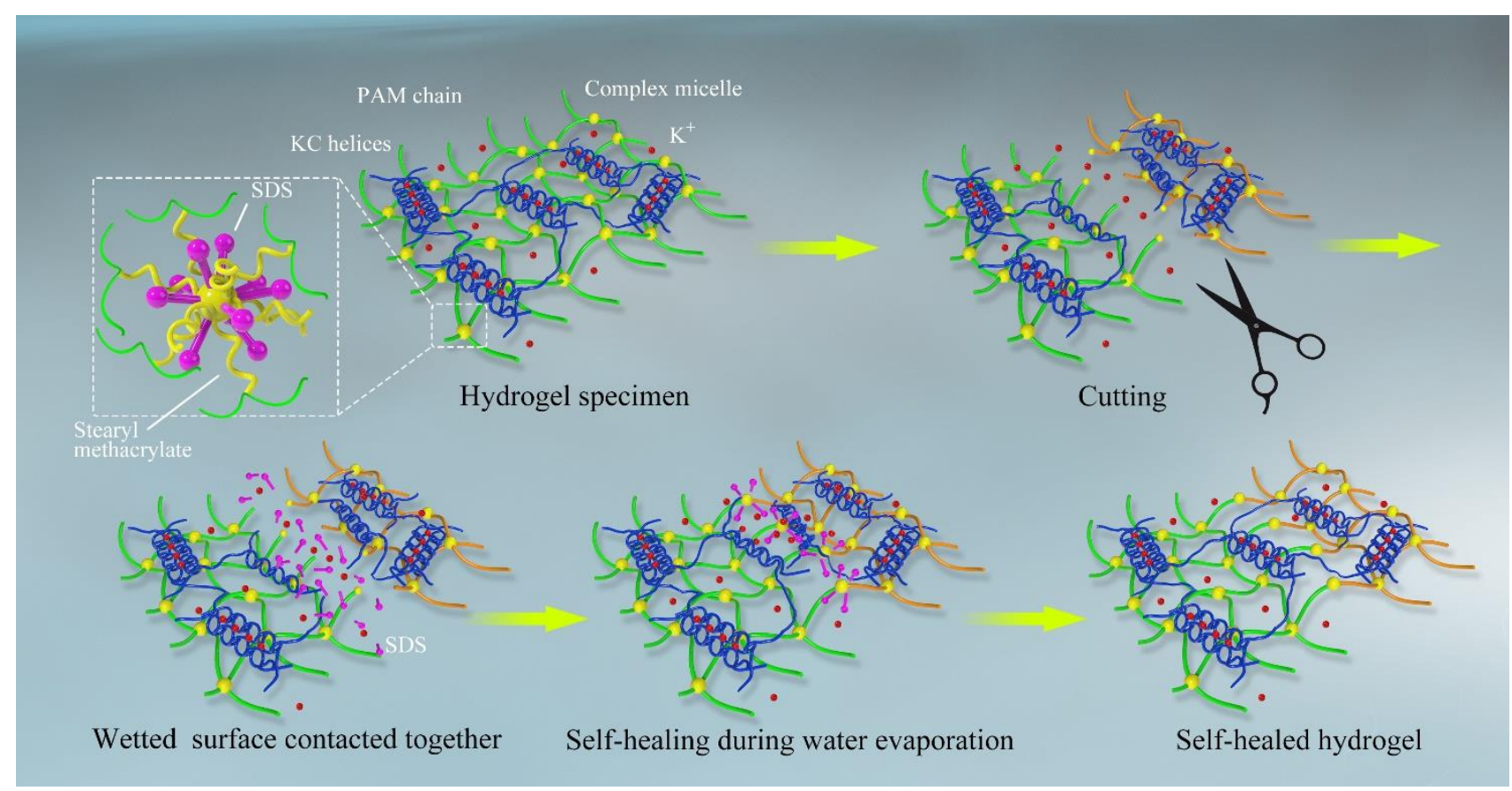

Figure 7. Schematic drawing of the potential self-healing mechanism of the KC/PAM DPC-DN hydrogels.

\subsection{Biocompatibility of KC/PAM DPC-DC Hydrogels}

The practical applications of DPC-DN hydrogels as artificial cartilage or diaphragm require 
excellent cytocompatibility of the materials. Figure 8a-e show the typical plan view of the rBMSCs morphologies on pristine PAM and KC/PAM hydrogel samples on day 1 . Only sporadic cells with a spherical morphology are found attached on the surface of the pristine PAM and KC/PAM-2.5 hydrogels, see Figur 8 (a-b). rBMSCs on KC/PAM hydrogels containing higher $\mathrm{KC}$ concentration show a spindle shape with a cytoplasmic and desirable extended morphology, confirming their good attachment and spreading, see Figure 8 (c-e). Compared to the pristine PAM and KC/PAM2.5 samples, a greater number of $\mathrm{rBMSCs}$ adhere onto the $\mathrm{KC} / \mathrm{PAM}-10$ hydrogel surface, and CLSM results from F-actin/Dapi counterstaining (Figure S5) further support the SEM results. With enhanced cell adhesion, cells grown in the KC/PAM-10 group has more extended filopodia and spread better with visible mature F-actin intracellular nanofibers. It has been proven that $\mathrm{KC}$ can positively influence the adhesion and reproduction of human mesenchymal stem cells (hMSCs) and human pluripotent stem cells (hPSCs). ${ }^{51-52}$ It also possesses chondrogenic potential for human adipose stem cells. Figure $8 \mathrm{f}$ quantitatively reveals the in vitro cellular viability on the pristine PAM and $\mathrm{KC} / \mathrm{PAM}$ hydrogels in $\alpha$-MEM for 1,3 , and 5 days, respectively. There are no statistical differences in cell viability between the tested groups on day 1 . At day 5 , a clear trend can be seen where the cell viability increases with increasing $\mathrm{KC}$ concentration in the hydrogels, and $\mathrm{KC} / \mathrm{PAM}$ 7.5 and KC/PAM-10 show the best cell viability. The data suggests that the incorporation of KC has successfully improved the cytocompatibility of PAM. 

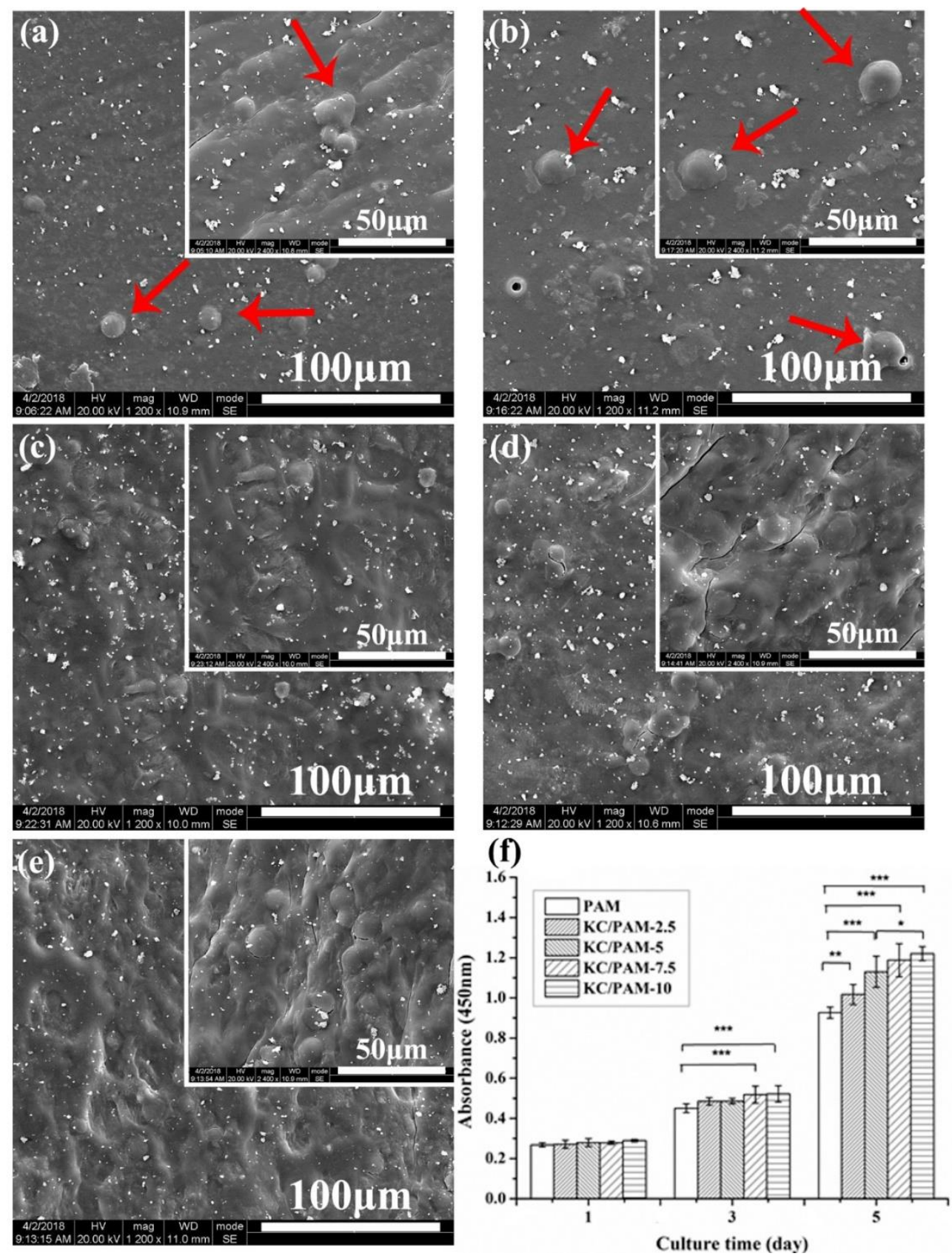

Figure 8. SEM images for rBMSCs morphologies on the (a) pristine PAM, (b) KC/PAM-2.5, (c) KC/PAM-5, (d) KC/PAM-7.5, (e) KC/PAM-10 DPC-DN hydrogels. (f) Cell proliferation of rBMSCs on the surface of the pristine PAM and KC/PAM hydrogels. Red arrows point to the round cells. 


\section{CONCLUSION}

In summary, we have successfully fabricated KC/PAM DN hydrogels through dual physically crosslinking. The KC/PAM hydrogels consist of hydrophobically associated PAM as the soft, stretchable first network, and monovalent cation $\left(\mathrm{K}^{+}\right)$linked $\mathrm{KC}$ as the stiff, brittle second network. The synergetic interaction between the two networks within the hydrogels has led to their significantly enhanced mechanical properties (tensile strength as high as $1320 \pm 46 \mathrm{kPa}$ and toughness as high as $6900 \pm 280 \mathrm{~kJ} / \mathrm{m}^{3}$ ). Furthermore, the robust KC/PAM hydrogels show excellent self-recovery, self-healing capabilities, as well as appealing biocompatibility. As a result, the strategy of incorporating multiple noncovalent interactions to toughen DN hydrogels may enable their practical applications in challenging areas such as artificial diaphragm, tendon, and cartilage.

\section{ASSOCIATED CONTENT}

\section{Supporting Information}

Pore size distribution for pristine $\mathrm{KC}$, PAM, KC/PAM-2.5, KC/PAM-5, KC/PAM-7.5, and KC/PAM-10 hydrogels (Figure S1); Equilibrium water content and swelling ratio of KC/PAM series hydrogels (Figure S2); Thermogravimetric analysis and thermal decomposition rate curves of the pristine KC, PAM and KC/PAM-10 hydrogels (Figure S3); Dependence of storage modulus $\mathrm{G}^{\prime}$ and loss modulus G' during the temperature sweeping from $25^{\circ} \mathrm{C}$ to $70{ }^{\circ} \mathrm{C}$ at a heating step of $2^{\circ} \mathrm{C} / \mathrm{min}$ (Figure S4); CLSM images of the cell adhesion morphology and actin cytoskeleton of rBMSCs cultured on the pristine PAM, KC/PAM-5, KC/PAM-10 hydrogels films for 1 day (Figure 
S5); Healing efficiency of PAM, KC/PAM-2.5, and KC/PAM-10 hydrogels at different temperatures for 2 to $24 \mathrm{~h}$ (Table S1).

\section{AUTHOR INFORMATION}

\section{Corresponding Authors}

Li Zhang:*E-mail: nic1976@scu.edu.cn; zhangli9111@126.com

Weizhong Yang: *E-mail: ywz@scu.edu.cn

\section{Author Contributions}

The manuscript was written through the contributions of all authors. All authors have approved the final version of the manuscript. ${ }^{\wedge}$ These authors contributed equally.

\section{Funding Sources}

This work is funded by the National Natural Science Foundation of China $(51673131,81801848)$,

National Key Research and Development Program of China (2016YFA0201703/2016YFA0201700), Key Laboratory Open Project Fund of Hainan University (2018008), and the Sichuan Science and Technology Program (2017FZ0046, 2018JZ0026).

\section{Notes}

The authors declare no competing financial interest.

\section{References}

(1) Seliktar, D. Designing Cell-Compatible Hydrogels for Biomedical Applications. Science 2012, 336 (6085), 1124-1128.

(2) Li, J.; Mooney, D. J. Designing Hydrogels for Controlled Drug Delivery. Nat. Rev. Mater. 2016, 
$1(12), 16071$.

(3) Slaughter, B. V.; Khurshid, S. S.; Fisher, O. Z.; Khademhosseini, A.; Peppas, N. A. Hydrogels in Regenerative Medicine. Adv. Mater. 2010, 21 (32-33), 3307-3329.

(4) Brown, T. E.; Anseth, K. S. Spatiotemporal Hydrogel Biomaterials for Regenerative Medicine. Chem. Soc. Rev. 2017, 46 (21), 6532-6552.

(5) Sun, J. Y.; Keplinger, C.; Whitesides, G. M.; Suo, Z. Ionic Skin. Adv. Mater. 2015, 26 (45), 7608-7614.

(6) Massad-Ivanir, N.; Shtenberg, G.; Zeidman, T.; Segal, E. Construction and Characterization of Porous $\mathrm{SiO}_{2} /$ Hydrogel Hybrids as Optical Biosensors for Rapid Detection of Bacteria. Adv. Funct. Mater. 2010, 20 (14), 2269-2277.

(7) Morales, M. E.; Gallardo, V.; Clarés, B.; García, M. B.; Ruiz, M. A. Study and Description of Hydrogels and Organogels as Vehicles for Cosmetic Active Ingredients. Int. J. Cosmet. Sci. 2010, $32(4), 314-314$.

(8) Peng, X. W.; Zhong, L. X.; Ren, J. L.; Sun, R. C. Highly Effective Adsorption of Heavy Metal Ions from Aqueous Solutions by Macroporous Xylan-Rich Hemicelluloses-Based Hydrogel. J. Agr. Food. Chem. 2012, 60 (15), 3909-3916.

(9) Zhang, Z.; Zhang, R.; Decker, E. A.; Mcclements, D. J. Development of Food-Grade Filled Hydrogels for Oral Delivery of Lipophilic Active Ingredients: pH-Triggered Release. Food. Hydrocolloid. 2015, 44 (44), 345-352.

(10) Gong, J. P.; Katsuyama, Y.; Kurokawa, T.; Osada, Y. Double-Network Hydrogels with Extremely High Mechanical Strength. Adv. Mater. 2003, 15 (14), 1155-1158.

(11) Nakayama, A.; Kakugo, A.; Gong, J. P.; Osada, Y.; Takai, M.; Erata, T.; Kawano, S. High Mechanical Strength Double-Network Hydrogel with Bacterial Cellulose. Adv. Funct. Mater. 2004, 
14 (11), 1124-1128.

(12) Chen, Q.; Zhu, L.; Zhao, C.; Wang, Q.; Zheng, J. A Robust, One-Pot Synthesis of Highly Mechanical and Recoverable Double Network Hydrogels Using Thermoreversible Sol-Gel Polysaccharide. Adv. Mater. 2013, 25 (30), 4171-4176.

(13) Sun, J. Y.; Zhao, X.; Illeperuma, W. R. K.; Chaudhuri, O.; Oh, K. H.; Mooney, D. J.; Vlassak, J. J.; Suo, Z. Highly Stretchable and Tough Hydrogels. Nature 2012, 489 (7414), 133-136.

(14) Jang, S. S.; Rd, G. W.; Kalani, M. Y. Mechanical and Transport Properties of the Poly(ethylene oxide)-Poly(acrylic acid) Double Network Hydrogel from Molecular Dynamic Simulations. $J$. Phys. Chem.B. 2007, 111 (7), 1729-1737.

(15) Shin, H.; Olsen, B. D.; Khademhosseini, A. The Mechanical Properties and Cytotoxicity of Cell-Laden Double-Network Hydrogels Based on Photocrosslinkable Gelatin and Gellan Gum Biomacromolecules. Biomaterials 2012, 33 (11), 3143-3152.

(16) Gong, J. P. Materials both Tough and Soft. Science. 2014, 344 (6180), 161-162.

(17) Gong, J. P. Why Are Double Network Hydrogels So Tough? Soft. Matter. 2010, 6 (12), 2583 2590.

(18) Yuan, N.; Xu, L.; Wang, H.; Fu, Y.; Zhang, Z.; Liu, L.; Wang, C.; Zhao, J.; Rong, J. Dual Physically Cross-Linked Double Network Hydrogels with High Mechanical Strength, Fatigue Resistance, Notch-Insensitivity, and Self-Healing Properties. ACS Appl. Mater Interfaces 2016, 8 (49), 34034-34044.

(19) Tuncaboylu, D. C.; Argun, A.; Sahin, M.; Sari, M.; Okay, O. Structure Optimization of SelfHealing Hydrogels Formed via Hydrophobic Interactions. Polymer 2012, 53 (24), 5513-5522.

(20) Burattini, S.; Colquhoun, H. M.; Fox, J. D.; Friedmann, D.; Greenland, B. W.; Harris, P. J.; Hayes, W.; Mackay, M. E.; Rowan, S. J. A Self-Repairing, Supramolecular Polymer System: 
Healability as a Consequence of Donor-Acceptor pi-pi Stacking Interactions. Chem. Commun. 2009, 44 (44), 6717-6719.

(21) Gao, H.; Yao, H. Shape insensitive optimal adhesion of nanoscale fibrillar structures. P. Natl. Acad. Sci. USA. 2004, 101 (21), 7851-7856.

(22) Nakahata, M.; Takashima, Y.; Yamaguchi, H.; Harada, A. Redox-Responsive Self-Healing Materials Formed from Host-Guest Polymers. Nat. Commun. 2011, 2 (1), 511.

(23) Cordier, P.; Tournilhac, F.; Souliéziakovic, C.; Leibler, L. Self-Healing and Thermoreversible Rubber from Supramolecular Assembly. Nature 2008, 451 (7181), 977-980.

(24) Gao, H.; Wang, N.; Hu, X.; Nan, W.; Han, Y.; Liu, W. Double Hydrogen-Bonding pHSensitive Hydrogels Retaining High-Strengths Over a Wide pH Range. Macromol. Rapid. Comm. 2013, $34(1), 63-68$.

(25) Chen, Q.; Zhu, L.; Chen, H.; Yan, H.; Huang, L.; Yang, J.; Zheng, J. A Novel Design Strategy for Fully Physically Linked Double Network Hydrogels with Tough, Fatigue Resistant, and SelfHealing Properties. Adv. Funct. Mater. 2015, 25 (10), 1598-1607.

(26) Yang, C. H.; Wang, M. X.; Haider, H.; Yang, J. H.; Sun, J. Y.; Chen, Y. M.; Zhou, J.; Suo, Z. Strengthening Alginate/Polyacrylamide Hydrogels using Various Multivalent Cations. ACS Appl. Mater. Interfaces 2013, 5 (21), 10418-10422.

(27) Yuan, N.; Xu, L.; Zhang, L.; Ye, H.; Zhao, J.; Liu, Z.; Rong, J. Superior Hybrid Hydrogels of Polyacrylamide Enhanced by Bacterial Cellulose Nanofiber Clusters. Mater. Sci. Eng. C Mater. Biol. Appl. 2016, 67, 221-230.

(28) Sabzi, M.; Samadi, N.; Abbasi, F.; Mahdavinia, G. R.; Babaahmadi, M. Bioinspired Fully Physically Cross-Linked Double Network Hydrogels with A Robust, Tough and Self-Healing Structure. Mater. Sci. Eng. C Mater. Biol. Appl. 2017, 74, 374-381, 
(29) Nakajima, T.; Kurokawa, T.; Ahmed, S.; Wu, W. L.; Gong, J. P. Characterization of Internal Fracture Process of Double Network Hydrogels under Uniaxial Elongation. Soft. Matter. 2013, 9 (6), 1955-1966.

(30) Wang, X.-H.; Song, F.; Qian, D.; He, Y.-D.; Nie, W.-C.; Wang, X.-L.; Wang, Y.-Z. Strong and Tough Fully Physically Crosslinked Double Network Hydrogels with Tunable Mechanics and High Self-Healing Performance. Chem. Eng. J. 2018, 349, 588-594.

(31) Gong, Z.; Zhang, G.; Zeng, X.; Li, J.; Li, G.; Huang, W.; Sun, R.; Wong, C. High-Strength, Tough, Fatigue Resistant, and Self-Healing Hydrogel Based on Dual Physically Cross-Linked Network. ACS Appl. Mater. Interfaces 2016, 8 (36), 24030-24037.

(32) Liu, S.; Li, L. Recoverable and Self-healing Double-Network Hydrogel based on $\kappa-$ Carrageenan. ACS Appl. Mater. Interfaces 2016, 8 (43), 29749-29758

(33) And, M. T.; Chiba, A.; Date, M. Gelation Mechanism of $\kappa-$ and ı-Carrageenan Investigated by Correlation between the Strain-Optical Coefficient and the Dynamic Shear Modulus. Macromolecules. 2001, 34 (21), 7427-7434.

(34) Yuguchi, Y.; Urakawa, H.; Kajiwara, K. Structural Characteristics of Carrageenan Gels: Various Types of Counter Ions. Food. Hydrocolloid. 2003, 17 (4), 481-485.

(35) Stone, A. K.; Nickerson, M. T. Formation and Functionality of Whey Protein Isolate-(Kappa-, Iota-, and Lambda-Type) Carrageenan Electrostatic Ccomplexes. Food. Hydrocolloid. 2012, 27 (2), 271-277.

(36) Yuan, H.; Song, J.; Li, X.; Li, N.; Dai, J. Immunomodulation and Antitumor Activity of $\kappa-$ Carrageenan Oligosaccharides. Cancer. Lett. 2006, 243 (2), 228-234.

(37) Nourmohammadi, J.; Roshanfar, F.; Farokhi, M.; Nazarpak, M. H. Silk Fibroin/KappaCarrageenan Composite Scaffolds with Enhanced Biomimetic Mineralization for Bone 
Regeneration Applications. Mater. Sci. Eng. C Mater. Biol. Appl. 2017, 76, 951-958.

(38) Mihaila, S. M.; Gaharwar, A. K.; Reis, R. L.; Marques, A. P.; Gomes, M. E.; Khademhosseini, A. Photocrosslinkable Kappa-Carrageenan Hydrogels for Tissue Engineering Applications. $A d v$. Healthc. Mater. 2013, 2 (6), 895-907.

(39) Popa, E. G.; Caridade, S. G.; Mano, J. F.; Reis, R. L.; Gomes, M. E. Chondrogenic Potential of Injectable $\kappa$-Carrageenan Hydrogel with Encapsulated Adipose Stem Cells for Cartilage TissueEngineering Applications. J. Tissue. Eng. Regen. Med. 2015, 9 (5), 550-563.

(40) Li, L.; Ni, R.; Shao, Y.; Mao, S. Carrageenan and Its Applications in Drug Delivery. Carbohyd. Polym. 2014, 103 (1), 1-11.

(41) Mahdavinia, G. R.; Etemadi, H.; Soleymani, F. Magnetic/pH-Responsive Beads Based on Caboxymethyl Chitosan and к-Carrageenan and Controlled Drug Release. Carbohyd. Polym. 2015, $128,112-121$.

(42) Yegappan, R.; Selvaprithiviraj, V.; Amirthalingam, S.; Jayakumar, R. Carrageenan Based Hydrogels for Drug Delivery, Tissue Engineering and Wound Healing. Carbohyd. Polym. 2018, $198,385-400$

(43) Hou, Y.; Liu, K.; Tu, Y.; Zhang, L.; Li, Y.; Chen, C. Preparation of PVA Hydrogel with HighTransparence and Investigations of Its Transparent Mechanism. RSC Adv. 2015, 5 (31), 2402324030.

(44) Lu, X.; Chan, C. Y.; Lee, K. I.; Ng, P. F.; Fei, B.; Xin, J. H.; Fu, J. Super-Tough and ThermoHealable hydrogel — Promising for Shape-Memory Absorbent Fiber. J. Mater. Chem. B 2014, 2 (43), 7631-7638.

(45) Pourjavadi, A.; Harzandi, A. M.; Hosseinzadeh, H. Modified Carrageenan 3. Synthesis of A Novel Polysaccharide-Based Superabsorbent Hydrogel via Graft Copolymerization of Acrylic 
Acid onto Kappa-Carrageenan in Air. Eur. Polym. J. 2004, 40 (7), 1363-1370.

(46) Bueno, V. B.; Takahashi, S. H.; Catalani, L. H.; de Torresi, S. I.; Petri, D. F. Biocompatible Xanthan/Polypyrrole Scaffolds for Tissue Engineering. Mater. Sci. Eng. C Mater. Biol. Appl. 2015, $52,121-128$.

(47) Minsk, L. M.; Kotlarchik, C.; Meyer, G. N.; Kenyon, W. O. Imidization During Polymerization of Acrylamide. J. Polym. Sci. Pol. Chem. 2010, 12 (1), 133-140.

(48) Burrows, H. D.; Ellis, H. A.; Utah, S. I. Adsorbed metal ions as stabilizers for the thermal degradation of polyacrylamide. Polymer 1981, 22 (12), 1740-1744.

(49) Yasuda, K.; Ping Gong, J.; Katsuyama, Y.; Nakayama, A.; Tanabe, Y.; Kondo, E.; Ueno, M.;

Osada, Y. Biomechanical Properties of High-Toughness Double Network Hydrogels. Biomaterials 2005, $26(21), 4468-4475$.

(50) Luo, F.; Sun, T. L.; Nakajima, T.; Kurokawa, T.; Zhao, Y.; Ihsan, A. B.; Guo, H. L.; Li, X. F.; Gong, J. P. Crack Blunting and Advancing Behaviors of Tough and Self-healing Polyampholyte Hydrogel. Macromolecules 2014, 47 (17), 6037-6046.

(51) Vignesh, S.; Gopalakrishnan, A.; M.R, P.; Nair, S. V.; Jayakumar, R.; Mony, U. Fabrication of Micropatterned Alginate-Gelatin and k-Carrageenan Hydrogels of Defined Shapes Using Simple Wax Mould Method as a Platform for Stem Cell/Induced Pluripotent Stem Cells (iPSC) Culture. Int. J. Biol. Macromol. 2018, 112, 737-744.

(52) Goonoo, N.; Khanbabaee, B.; Steuber, M.; Bhawluximon, A.; Jonas, U.; Pietsch, U.; Jhurry, D.; Schönherr, H. $\kappa$-Carrageenan Enhances the Biomineralization and Osteogenic Differentiation of Electrospun PHB and PHBV Fibers. Biomacromolecules 2017, 18 (5),1563-1573. 


\section{TOC}
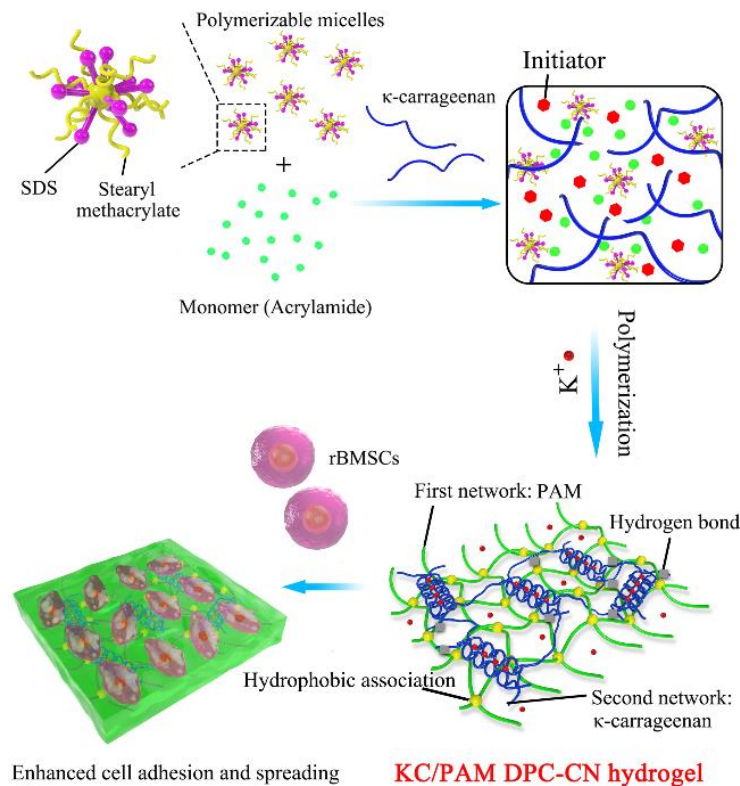